\title{
Design and vertical tests of double-quarter wave cavity prototypes for the high-luminosity LHC crab cavity system
}

\author{
S. Verdú-Andrés, ${ }^{1, *}$ K. Artoos, ${ }^{2}$ S. Belomestnykh,${ }^{1,3,4}$ I. Ben-Zvi, ${ }^{1,4}$ C. Boulware, ${ }^{5}$ G. Burt, ${ }^{6,7}$ \\ R. Calaga, ${ }^{2}$ O. Capatina, ${ }^{2}$ F. Carra, ${ }^{2}$ A. Castilla, ${ }^{2}$ W. Clemens, ${ }^{8}$ T. Grimm,${ }^{5}$ N. Kuder, ${ }^{2}$ R. Leuxe, ${ }^{2}$ \\ Z. Li, ${ }^{9}$ E. A. McEwen, ${ }^{8}$ H. Park, ${ }^{8}$ T. Powers,${ }^{8}$ A. Ratti, ${ }^{9,10}$ N. Shipman,${ }^{6,7}$ J. Skaritka, ${ }^{1}$ Q. Wu, ${ }^{1}$ \\ B. P. Xiao, ${ }^{1}$ J. Yancey, ${ }^{5}$ and C. Zanoni ${ }^{2, \dagger}$ \\ ${ }^{1}$ Brookhaven National Laboratory (BNL), Upton, New York 11973, USA \\ ${ }^{2}$ European Organization for Nuclear Research CERN, 1217 Meyrin, Switzerland \\ ${ }^{3}$ Fermi National Laboratory, Batavia, Illinois 60510, USA \\ ${ }^{4}$ Stony Brook University, Stony Brook, New York 11790, USA \\ ${ }^{5}$ Niowave Inc., East Lansing, Michigan 48906, USA \\ ${ }^{6}$ Lancaster University, Bailrigg, Lancaster LA1 4YW, United Kingdom \\ ${ }^{7}$ Cockcroft Institute, Daresbury, Warrington WA4 4AD, United Kingdom \\ ${ }^{8}$ Jefferson Lab, Newport News, Virginia 23606, USA \\ ${ }^{9}$ SLAC National Accelerator Laboratory, Palo Alto, California 94025, USA \\ ${ }^{10}$ Lawrence Berkeley National Laboratory, Berkeley, California 94720, USA
}

(Received 21 May 2018; published 13 August 2018)

\begin{abstract}
Crab crossing is essential for high-luminosity colliders. The high-luminosity Large Hadron Collider (HL-LHC) will equip one of its interaction points (IP1) with double-quarter wave (DQW) crab cavities. A DQW cavity is a new generation of deflecting rf cavities that stands out for its compactness and broad frequency separation between fundamental and first high-order modes. The deflecting kick is provided by its fundamental mode. Each HL-LHC DQW cavity shall provide a nominal deflecting voltage of 3.4 MV, although up to 5.0 MV may be required. A proof-of-principle (POP) DQW cavity was limited by quench at 4.6 MV. This paper describes a new, highly optimized cavity, designated the DQW SPS series, which satisfies dimensional, cryogenic, manufacturing, and impedance requirements for beam tests at the Super Proton Synchrotron (SPS) and operation in the LHC. Two prototypes of this DQW SPS series were fabricated by U.S. industry and cold tested after following a conventional superconducting radio-frequency surface treatment. Both units outperformed the POP cavity, reaching a deflecting voltage of 5.3-5.9 MV. This voltage - the highest reached by a DQW cavity — is well beyond the nominal voltage of 3.4 MV and may even operate at the ultimate voltage of 5.0 MV with a sufficient margin. This paper covers fabrication, surface preparation, and cryogenic rf test results and implications.
\end{abstract}

DOI: $10.1103 /$ PhysRevAccelBeams.21.082002

\section{INTRODUCTION}

Crab crossing is an essential mechanism for highluminosity colliders. The high-luminosity Large Hadron Collider (HL-LHC) will implement crab crossing at the interaction point (IP) of ATLAS (IP1) and CMS (IP5) [1]. The crabbing system of HL-LHC will follow the local scheme [1]. The IP1 will be equipped with a set of

"Corresponding author.

sverdu@bnl.gov

Present address: European Southern Observatory (ESO), Munich, Germany.

Published by the American Physical Society under the terms of the Creative Commons Attribution 4.0 International license. Further distribution of this work must maintain attribution to the author(s) and the published article's title, journal citation, and DOI. double-quarter wave (DQW) cavities [2], while IP5 will have a set of rf dipole (RFD) cavities [3,4]. The four-rod crab cavity [5] was also considered for the HL-LHC crabbing system and then downselected in favor of the DQW and RFD cavities. The HL-LHC crab cavities are superconducting radio-frequency (SRF) bulk niobium cavities and operate at $400 \mathrm{MHz}$ in continuous wave (cw) mode [6]. Crab crossing also appears in the baseline design of electron-ion colliders eRHIC and JLeIC. Both will require crab crossing to reach the necessary luminosity levels for relevant nuclear physics studies $[7,8]$. The eRHIC crab cavities are based on the DQW design developed for HL-LHC [9].

The operational experience of crab cavities with beam is currently limited to the electron-positron collider KEK-B [10]. Electron-positron beams are highly damped by synchrotron radiation and, thus, more robust against crabbing errors than proton machines. The KEK-B crab cavities 
were elliptical. With a higher-order crabbing mode, their size was too large to fit between the closely spaced beam pipes of the LHC. This led to the development of the compact DQW cavities. Before crabbing the proton bunches of LHC, a cryomodule with two fully dressed DQW cavities will be tested with a beam in SPS in 2018 to address crab cavity operational issues in a proton machine. Two DQW SPS-series cavity prototypes were fabricated in U.S. industry following conventional surface treatments for SRF cavities. The two DQW cavities and equipment used for the SPS tests were fabricated in-house by CERN [11]. All four cavities share the same rf design.

Deflecting of cavities are primarily TM-110-like structures [12-16]. The DQW cavity instead belongs to a generation of TEM-like, low-frequency, compact deflecting rf cavities [3,5,17]. The fundamental mode of a DQW cavity provides the necessary deflecting kick for bunch crabbing. The DQW cavity stands out for its compactness and broad frequency separation between fundamental and first high-order modes [18]. The DQW cavity can be seen as two coaxial quarter wave $(\mathrm{QW})$ resonators mirror symmetric with respect to their open-end plane. The opposing inner conductor poles behave as capacitor plates. The highest magnetic field region of the fundamental mode is found in the cavity dome (the shorted ends of the QW resonator), whereas the highest electric field region is in the capacitive plates [19]. The field distribution in the rest of the cavity body corresponds to a TEM-like mode. The voltage sustained between the plates provides a deflecting kick to the bunch. The accelerating voltage seen by a bunch traveling on axis through a symmetric DQW is zero [18].

The DQW cavity originated from a QW resonator [20]. The fundamental mode of the QW resonator provided the deflecting kick. This cavity was short in the direction of the opposing beam line, and the first higher-order mode (HOM) and fundamental mode were well separated [21]. A pedestal was later included to reduce the nonzero accelerating gradient on axis [22]. Finally, the cavity was symmetrized with respect to the $y=0$ plane (becoming a double-QW cavity) to completely suppress the accelerating gradient at the expense of reducing the mode separation between the fundamental mode and first HOM and becoming larger in the direction of the opposing beam line $[18,23]$. The DQW cavity has a heavy capacitive loading (approaching it to the reentrant cavity) which differentiates it from the classical half-wave resonator with larger inductive loading.

The current HL-LHC baseline accounts for a total of 16 crab cavities: two cavities per IP per side per beam [24]. The crab cavities of HL-LHC will be located between dipole D2 and quadrupole Q4 in the LHC interaction region (IR). In this location, the two beams are well separated into their corresponding individual pipes, and the betatron function is large enough to minimize the required crabbing voltage [1]. Each cavity will provide a nominal deflecting voltage of $3.4 \mathrm{MV}$, but up to $5.0 \mathrm{MV}$ may be required for full geometric overlap of the colliding bunches. The delivery of such high deflecting voltage requires operation at significantly high peak surface fields. A proof-ofprinciple (POP) DQW cavity was fabricated by Niowave Inc. in 2013 for $\mathrm{rf}$ performance validation. The POP cavity reached a peak surface magnetic field of $116 \mathrm{mT}$ before quenching at the maximum deflecting voltage of $4.6 \mathrm{MV}$ [25]. A new, highly optimized version-designated the DQW SPS series—satisfies dimensional, cryogenic, manufacturing, and impedance requirements for operation in the LHC. This new design also presents lower peak fields with the aim at reaching higher deflecting voltages than the POP cavity.

The present paper is organized as follows. The first part of the paper discusses the main design features of the SPS-series cavities and provides comparisons with the POP cavity. The second part describes the fabrication and surface treatment of two U.S. DQW SPS-series cavity prototypes built to test the cavity concept and steer the development of the SPS cavities at CERN. The third part presents bare cavity cold test results and discusses cryogenic rf performances of these prototypes. Fabrication and test results of the CERN cavities are out of the scope of this paper.

\section{SPS-SERIES DQW CAVITY DESIGN}

\section{A. rf design}

The SPS-series DQW cavity presents three main important new features. First of all, the design is compatible with both vertical and horizontal kick configurations. Crab crossing will be implemented for two LHC IPs: IP1 (ATLAS) and IP5 (CMS). The beams cross in the vertical plane at IP1 and in the horizontal plane at IP5. The second beam pipe of the LHC limits the cavity width (when used in the vertical kick configuration) and height (when used in the horizontal kick configuration) [26]. Similarly to the POP cavity design, the cavity adopts a pronounced hourglass shape to accommodate the second beam pipe in IP1. In addition, the cavity takes an elliptical racetrack profile for frequency tuning, as the cavity height is fixed by the second beam pipe in IP5. The integration of the DQW cavity into the cryomodule was simpler when the DQW provided the vertical deflecting kick. Even if the DQW cavity was finally selected to provide the vertical deflecting kick, the main cavity body still had to meet the dimensional requirements imposed by the second beam pipe to allow its use for either vertical or horizontal crabbing in the LHC. Figure 1 shows the DQW crab cavity for both the vertical and horizontal kick configurations. The slimmer section is usually referred to as the cavity waist.

Second, the rf coupling ports are large enough to incorporate a $40 \mathrm{~kW}$ fundamental power coupler (FPC) 


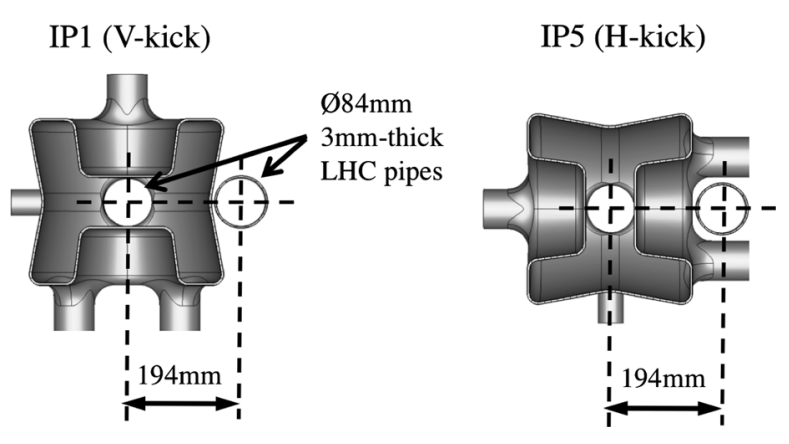

FIG. 1. The SPS-series DQW cavity satisfies the LHC geometric restrictions to provide a deflecting kick in both the vertical and horizontal kick configurations.

and ensure the extraction of $1 \mathrm{~kW}$ HOM power with a minimum number of ports [27] to meet the rf requirements [1]. In total, the cavity has four 62 -mm-diameter ports. One port serves the FPC; the other three host the HOM filters. To ease cleaning of the cavity's interior, these four ports are located in the cavity's dome-the inductive region of the cavity. For efficient coupling to the magnetic field, hooktype coupling elements are used for both the FPC and HOM filters. The hook also provides good coupling to the two lowest HOMs, found at 567 and $588 \mathrm{MHz}$, both with a high magnetic field concentrated in the cavity dome. All couplers are detachable from the cavity to facilitate cleaning and installation. The elliptical racetrack has a constant width to accommodate the port openings. The selected port configuration, shown in Fig. 2, provides the lowest external $Q$ of HOMs for frequencies of up to $2 \mathrm{GHz}$ [28]. Modes with frequencies above $2 \mathrm{GHz}$ are expected to be Landau damped [26].

Two ports are in line with the beam axis; the other two are at an angle of $45^{\circ}$ with respect to the beam axis to allow the passage of the second beam pipe of LHC when the cavity is used to provide a horizontal kick. The port distribution asymmetry introduces (i) a nonzero accelerating voltage of $15 \mathrm{kV}$ for a nominal deflecting voltage of 3.4 MV (negligible when compared to the LHC energy beam) and (ii) an electric field center offset of $0.23 \mathrm{~mm}$ displaced towards the cavity bottom, which falls within the alignment tolerances [26].

The pickup port, opened on one of the beam pipes to preserve the cavity's symmetry, is perpendicular to the deflecting kick direction to ease cavity manufacturing. A DQW SPS-series cavity dressed with three HOM filters and the pickup meets the impedance budget imposed for operation in the LHC [29]. Detailed discussion of the design, fabrication, and performance of the HOM filters and pickup will be addressed in a separate communication.

Finally, the port-cavity interface is optimized to reduce the magnetic peak field [30]. The magnetic field finds its maximum close to the port apertures in the cavity body. For a bare cavity with no port openings, a maximum peak magnetic field of $66 \mathrm{mT}$ (for a nominal deflecting voltage
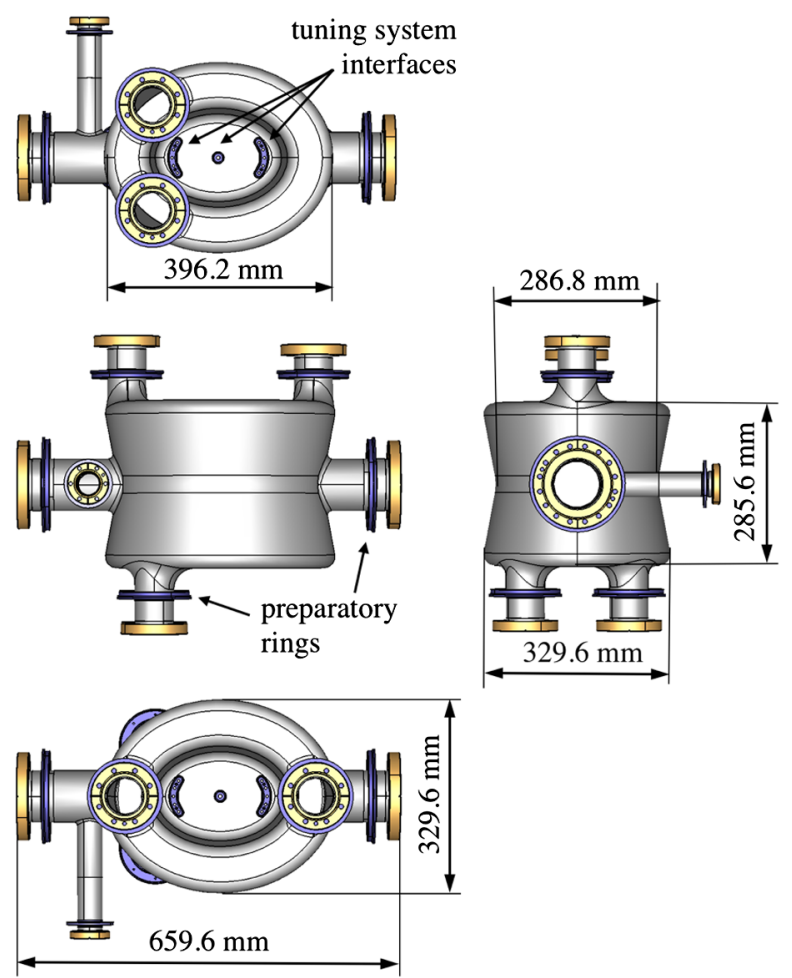

FIG. 2. Main dimensions at warm of the SPS-series DQW cavity mechanical design.

of $3.4 \mathrm{MV}$ ) is found along the inner radius of the cavity dome. The design of the port-cavity interface aimed to achieve a geometry with a reduced field enhancement that was also easy to fabricate. The selected port-cavity interface leads to a maximum peak surface magnetic field of $73 \mathrm{mT}$ for a nominal deflecting voltage of $3.4 \mathrm{MV}$. The highest field is located between the $45^{\circ}$ ports at the inner radius of the cavity dome.

Table I summarizes the main geometry properties of the SPS-series DQW crab cavity. General cavity dimensions are displayed in Fig. 2. The main electromagnetic properties of the SPS-series DQW cavity are given in Table II. The shunt impedance of the deflecting mode $R_{t} / Q$ is defined following the accelerator convention as

$$
\frac{R_{t}}{Q}=\frac{V_{t}^{2}}{\omega U}
$$

where $V_{\mathrm{t}}$ is the deflecting voltage, $\omega$ is the angular frequency $(\omega=2 \pi f)$ and $U$ is the stored energy. Fig. 3 shows the electric and magnetic fields along the geometric center of a DQW cavity used for the evaluation of $V_{\mathrm{t}}$. Note that $R_{t} / Q$ is given in units of impedance, instead of impedance per unit length, as is typically the convention for transverse shunt impedance. The SPS-series DQW cavity has an $R_{t} / Q$ of $429.3 \Omega$ for the fundamental (crabbing) mode. The geometry factor $G$, defined as the product of quality factor $Q_{0}$ and surface resistance $R_{s}$, is $87 \Omega$. This is a low geometry factor compared to the typical TESLA-type 
TABLE I. General geometry dimensions of the SPS-series DQW cavity at an ambient temperature (includes the wall thickness).

\begin{tabular}{lcc}
\hline \hline Cavity length (flange to flange) & 659.60 & $\mathrm{~mm}$ \\
Cavity height (port to port) & 495.71 & $\mathrm{~mm}$ \\
Cavity width (no pickup) & 329.60 & $\mathrm{~mm}$ \\
Beam pipe inner diameter & 84 & $\mathrm{~mm}$ \\
FPC and HOM port inner diameter & 62 & $\mathrm{~mm}$ \\
\hline \hline
\end{tabular}

TABLE II. Electromagnetic properties of the SPS-series DQW cavity.

\begin{tabular}{lccc}
\hline \hline Fundamental frequency & $f^{(0)}$ & 400.79 & $\mathrm{MHz}$ \\
First HOM frequency & $f^{(1)}$ & 567 & $\mathrm{MHz}$ \\
$\quad$ (longitudinal mode) & & & \\
Transverse $R / Q$ & $R_{t} / Q$ & 429.3 & $\mathrm{Ohm}$ \\
Geometry factor $_{\text {Peak surface magnetic field }}^{\mathrm{a}}$ & $G$ & 87 & $\mathrm{Ohm}$ \\
Peak surface electric field $^{\mathrm{a}}$ & $B_{p}$ & 72.8 & $\mathrm{mT}$ \\
Residual accelerating voltage $^{\mathrm{a}}$ & $E_{p}$ & 37.6 & $\mathrm{MV} / \mathrm{m}$ \\
Electric field center offset $_{\text {acc }}$ & 15.23 & $\mathrm{kV}$ \\
\hline \hline
\end{tabular}

${ }^{\mathrm{a}}$ For a nominal deflecting voltage of $3.4 \mathrm{MV}$.

elliptical cavity $(G \sim 270 \Omega)$ [31]. Since the BCS surface resistance for niobium at $400 \mathrm{MHz}$ and $2 \mathrm{~K}$ is only $1 \mathrm{n} \Omega$, the residual surface resistance could have a significant impact on the final $Q_{0}$ that the cavity achieved. Typical residual resistance values for $\mathrm{Nb}$ cavities are on the order of 5-10 $\mathrm{n} \Omega$ [32]. The required $Q_{0}$ (and so $R_{s}$ ) is established considering a reasonable load to the cryogenic system during beam tests in the SPS and operation in the LHC. The functional specifications for the LHC crab cavities establish a maximum dynamic heat load per cavity of $5 \mathrm{~W}$ for nominal operation at $3.4 \mathrm{MV}$ [6] that translates into a maximum $R_{s}$ of $16 \mathrm{n} \Omega$ for the DQW cavity delivering a 3.4 MV deflecting kick.

\section{B. Multipacting}

The phenomenon of multipacting can be a serious obstacle for the normal operation of rf cavities. Multipacting currents absorb rf power, degrade the coupling between the power source and rf cavity, heat the impacted surfaces to the extent of causing thermal breakdown in SRF cavities, and even break ceramic windows.

Simulations were conducted with the particle tracking codes Track3P of ACE3P [33] and Particle Studio of CST [34] to identify the potential multipacting sites and find at which voltage levels multipacting may occur. The secondary emission yield (SEY) curve for baked niobium was used in our studies [35]. Figure 4 displays the predicted multipacting sites by ACE3P in a SPS DQW cavity. Multipacting bands are found in (i) the cavity waist for deflecting voltage levels below $0.5 \mathrm{MV}$, (ii) the blending of the cavity dome, between 2 and $3 \mathrm{MV}$ and between 4.0 and $4.5 \mathrm{MV}$, (iii) the

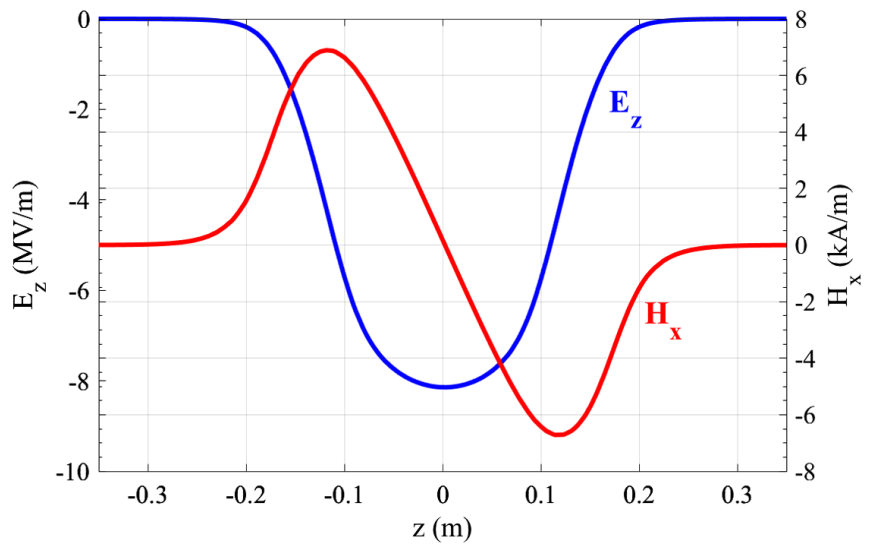

FIG. 3. Electric and magnetic field along the geometric center of a DQW cavity.

blending of the beam ports, between 1.6 and $4.0 \mathrm{MV}$, (iv) the blending of the small cavity ports, between 1.7 and $2.7 \mathrm{MV}$, and (v) the FPC port, below $0.5 \mathrm{MV}$. CST predicted similar multipacting bands and also identified a multipacting band between 1 and $2.5 \mathrm{MV}$ in the cavity waist.

Multipacting in the FPC port may require dedicated high-power conditioning. The maximum SEY of baked niobium is above 1 for electrons with an impact energy between 100 and $1500 \mathrm{eV}$, with the maximum SEY found at around $300 \mathrm{eV}$. Figure 5 shows the impact energy of multipacting electrons at different deflecting voltage levels. Multipacting in the HOM filters will be discussed in a future publication.

As a reference, we may consider the tests of the POP DQW cavity, with a structure similar to the SPS DQW cavity of this report. The POP DQW cavity exhibited

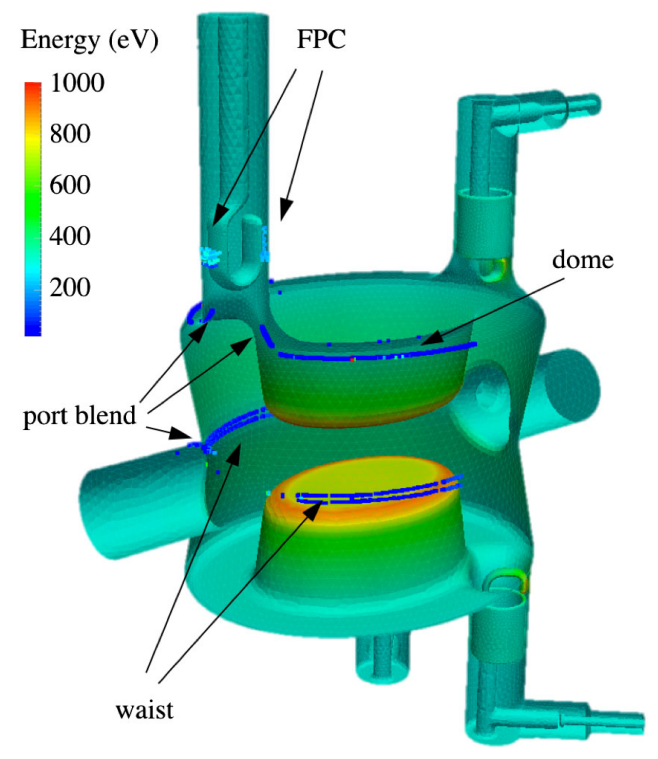

FIG. 4. Multipacting sites found in a DQW cavity by ACE3P Track3P. The color scale represents the impact energy in $\mathrm{eV}$ of multipacting electrons. 


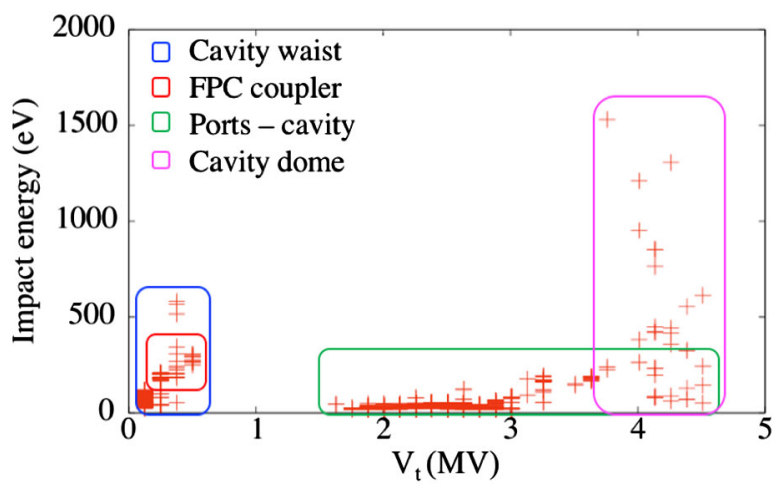

FIG. 5. Impact energy of multipacting electrons found by ACE3P Track3P simulations at different deflecting voltage levels.

multipacting below $0.5 \mathrm{MV}$ and between 2 and $3 \mathrm{MV}$. Those multipacting bands are also found for the SPS DQW cavity. Low field multipacting could be quickly processed through during the first test of the POP cavity and was not seen again in later cold tests at BNL [25].

\section{Mechanical design}

The position of the FPC port flange was chosen as a compromise between the losses in the rf-seal copper gasket and the conductive losses in the FPC tube. The length of the HOM ports is determined by the location of the capacitive cylinder in the HOM filter, which rejects most of the fundamental mode power back. The detailed HOM filter design is shown in Ref. [36]. The port is cut beyond the position of this capacitive cylinder. The largest power loss, $0.1 \mathrm{~W}$, is found for the rf-seal gasket in the shortest beam pipe. The loss in the rf-seal gaskets of the four $62-\mathrm{mm}$ diameter ports amounts only to $23 \mathrm{~mW}$. The loss in the FPC tube is below $1 \mathrm{~mW}$. All these values are computed for a nominal deflecting voltage of 3.4 MV.

The cavity prototypes include the interfaces to the helium vessel and tuning system. Figure 2 shows a bare DQW crab cavity with flanges, preparatory rings, and interfaces to the tuning system.

\section{CAVITY FABRICATION}

The fabrication model was prepared considering the volume changes due to buffered chemical polishing (BCP), cooldown, and weld shrinkage. The parts were deep drawn from fine grain $R R R \geq 300$ niobium sheets and then electron beam welded to form the three main subassemblies shown in Fig. 6. The main cavity body parts were fabricated out of 4-mm-thick sheets to withstand a pressure difference of 1.8 bar on the cavity walls (2.7 bar after application of the safety coefficient) before yielding [37]. As the extrusion of port nipples inevitably resulted in a local reduction of the material thickness, the cavity extremities were manufactured from 3-mm-thick sheets to connect with the extruded port nipples. The cavity ports were equipped
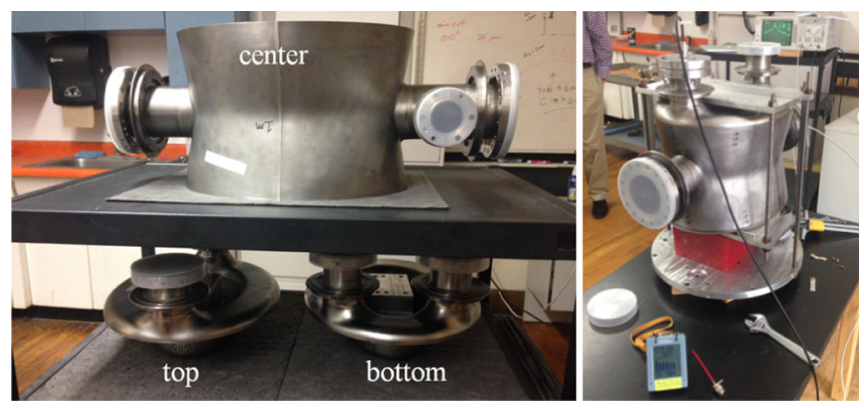

FIG. 6. (Left) Subassemblies for a DQW cavity. (Right) Clamped assembly of a DQW cavity.

with $90^{\circ}$ knife-edge $\mathrm{CF}$ flanges. All flanges were made from multidirectional (3D) forged, austenitic stainless-steel grade $316 \mathrm{LN}$. The stainless-steel flanges were vacuum brazed to the niobium tubes. The ports also incorporated $\mathrm{NbTi}$ adaptors to interface the niobium cavity with the titanium helium vessel [38].

The installed cavity, operating at $2 \mathrm{~K}$ and delivering $3.4 \mathrm{MV}$ to the $450 \mathrm{GeV}$ proton beam of SPS, will operate at $(400.79 \pm 0.06) \mathrm{MHz}$. The target frequency for the manufactured cavity was $400.29 \mathrm{MHz}$ at ambient conditions, after considering the frequency changes due to $\mathrm{BCP}$, coupler insertion, evacuation, and cooldown.

The first tuning of the cavities took place during cavity fabrication, when the cavity was still divided into three main subassemblies. The subassemblies were clamped together, and the frequency of the assembly was measured. Then, some material was trimmed out of the subassembly edges. This trimming operation does not change the length of the cavity poles; it changes only the distance between the capacitive plates. The consequent increase of capacitance translates into a reduction of the cavity frequency. The material removal was performed in several iterations to carefully approach the target frequency. For this purpose, the subassemblies were fabricated leaving additional material at their edges (overlength). An equal amount of material was trimmed at one side and the other of the beam axis to preserve the alignment of the electric field center. Figure 7 shows the frequency evolution for the clamped assembly of cavity 1 as material is removed from the subassembly edges. The manufactured cavity showed a slightly higher frequency than nominal given a certain overlength. Dimensional control of the fabricated subassemblies identified a smaller volume than modeled in the high-magnetic field region of the cavity, which explains the higher frequency of the manufactured cavity. Simulated and measured trim tuning sensitivity curves run parallel, evidencing the good agreement between the prediction and measurements.

Before the last trimming step, the wall was thinned down to $3 \mathrm{~mm}$ along the seams of the two last welds. The thinning intended to improve the matching of subassembly edges, ease weld execution, and reduce the weld penetration depth. This operation had already been implemented in the past for 


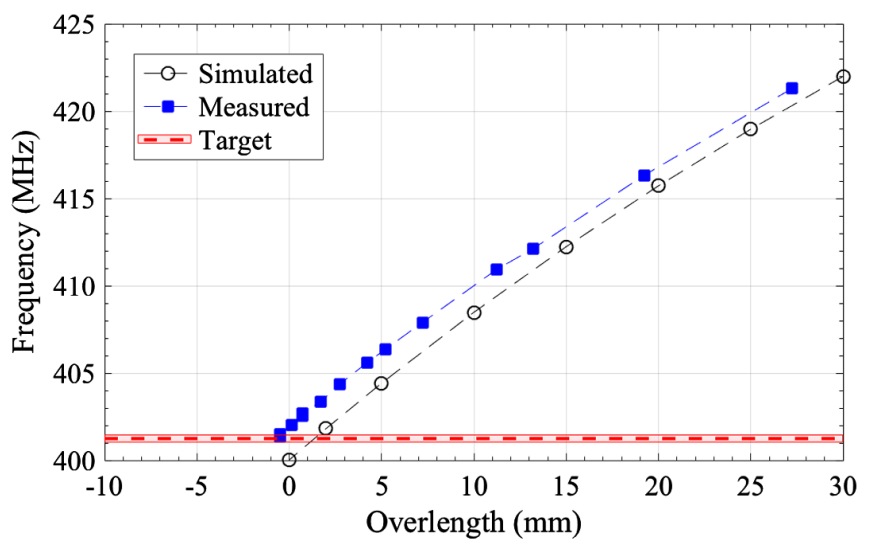

FIG. 7. Frequency evolution during trim tuning of cavity 1. Each data point (blue square) corresponds to the frequency measured at a certain trimming stage. The dashed red line represents the target frequency for the clamped assembly, 401.25 MHz, while the red shadowed area represents the frequency tolerance, $\pm 0.20 \mathrm{MHz}$. In black, the frequency response to trim tuning from CST simulations.

other cavities [39]. ANSYS simulations [40] found that this local thickness reduction should not compromise the structural integrity of the DQW cavity during cooldown [37]. A small amount of material was removed from the internal surface (or rf surface), while most of the material was removed from the outer surface. The interior surface was machined with a ball mill to ensure a smooth transition.

Upon reception at Jefferson Lab, the frequency of the clamped assembly was $401.35 \mathrm{MHz}$ for cavity 1 , within the accepted range of $(401.25 \pm 0.20) \mathrm{MHz}$. The trim tuning process demonstrated the required control for cavity production. The cavity subassemblies were then joined using electron beam welding at Jefferson Lab. The welding was expected to reduce the assembly frequency by almost $1 \mathrm{MHz}$. However, the welding process shifted the frequency of cavity 1 in the opposite direction, to $401.60 \mathrm{MHz}$. The frequency of cavity 2 also did not behave as expected upon welding. Later work on the CERN cavities found that the cavities were deformed by the two last welds. Such deformation had not been considered in the frequency shift estimation. A tuning method was implemented at CERN to revert the deformation and tune the cavities to the target frequency [41].

\section{SURFACE PREPARATION AND CAVITY ASSEMBLY}

Surface preparation and cold tests of the two cavities were conducted in the SRF facility of Jefferson Lab. The cavity surface was prepared according to the following procedure [42]: ultrasound bath degreasing, bulk BCP, 10 hr hydrogen degassing in UHV furnace at $600^{\circ} \mathrm{C}$, another ultrasound bath degreasing, light $\mathrm{BCP}$, manual rinsing of every port followed by high-pressure rinsing (HPR) in a dedicated HPR cabinet with ultrapure water (cavity in vertical orientation, sprinkler moving up and down along

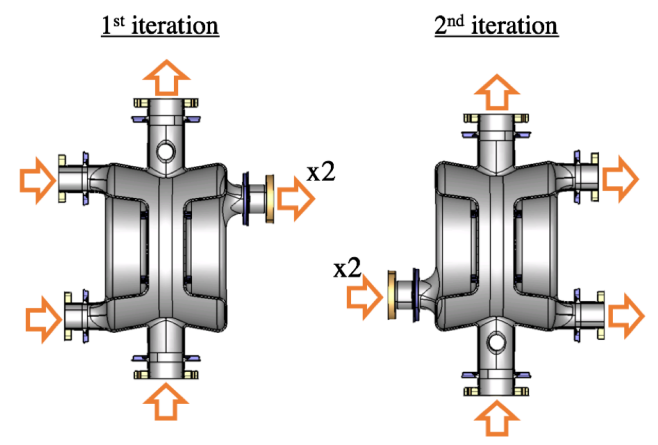

FIG. 8. Bulk BCP of a cavity in two iterations.

beam pipe axis), assembly in a class 10 (ISO 4) clean room, slow bleed pumping, leak check, and $120^{\circ} \mathrm{C}$ baking of the evacuated cavity in a furnace dedicated to SRF applications. Dimensional and frequency controls were done at different stages of the surface treatment. The wall thickness was measured in several locations before and after each $\mathrm{BCP}$ iteration. The cavity frequency was checked before and after bulk $\mathrm{BCP}, 600^{\circ} \mathrm{C}$ baking, and light $\mathrm{BCP}$.

The BCP treatment was conducted in a fixed bench using an acid mixture of $\mathrm{HF}$ (48\% concentration), $\mathrm{HNO}_{3}(70 \%$ concentration), and $\mathrm{H}_{3} \mathrm{PO}_{4}$ (85\% concentration) in a $1: 1: 2$ ratio. Bulk $\mathrm{BCP}$ was performed in two iterations to provide a more uniform material removal. Each iteration was conducted using different acid inlet and outlet ports and with the cavity in a different orientation, as depicted in Fig. 8. The bulk BCP performed on cavity 1 (2) removed an average of 260 (140) $\mu \mathrm{m}$ from the bottom subassembly and $180(160) \mu \mathrm{m}$ from the top subassembly.

The interior surface of cavity 1 looked smooth after bulk BCP, except for some of the features shown in Fig. 9: (i) pits in the center of both capacitive plates, (ii) some rough surface in the high electric field region of the capacitive plates, (iii) "orange peel" or pitting close to the HOM port of the top subassembly, and (iv) a rough bead in the last weld joining the center and bottom subassemblies, in the surroundings of the HOM port which was the closest to the pickup tube.

After $600^{\circ} \mathrm{C}$ baking, a light $\mathrm{BCP}$ of about $40 \mu \mathrm{m}$ was performed on the cavities in the same configuration of the first bulk BCP iteration. The cavities were rinsed inside the $\mathrm{BCP}$ cabinet multiple times with cold water and then warm water after each $\mathrm{BCP}$ iteration.

\section{BARE CAVITY COLD RF TESTS}

The two cavities were tested in the SRF Facility of Jefferson Lab. The design and location of test couplers was common to all the DQW SPS-series cavities developed in the U.S. and at CERN. The maximum power available for conditioning was limited to $200 \mathrm{~W}$. The input probe (fixed) was inserted into the short beam port to provide an external $Q$ of about $2 \times 10^{9}$. This value was (a) low enough for 


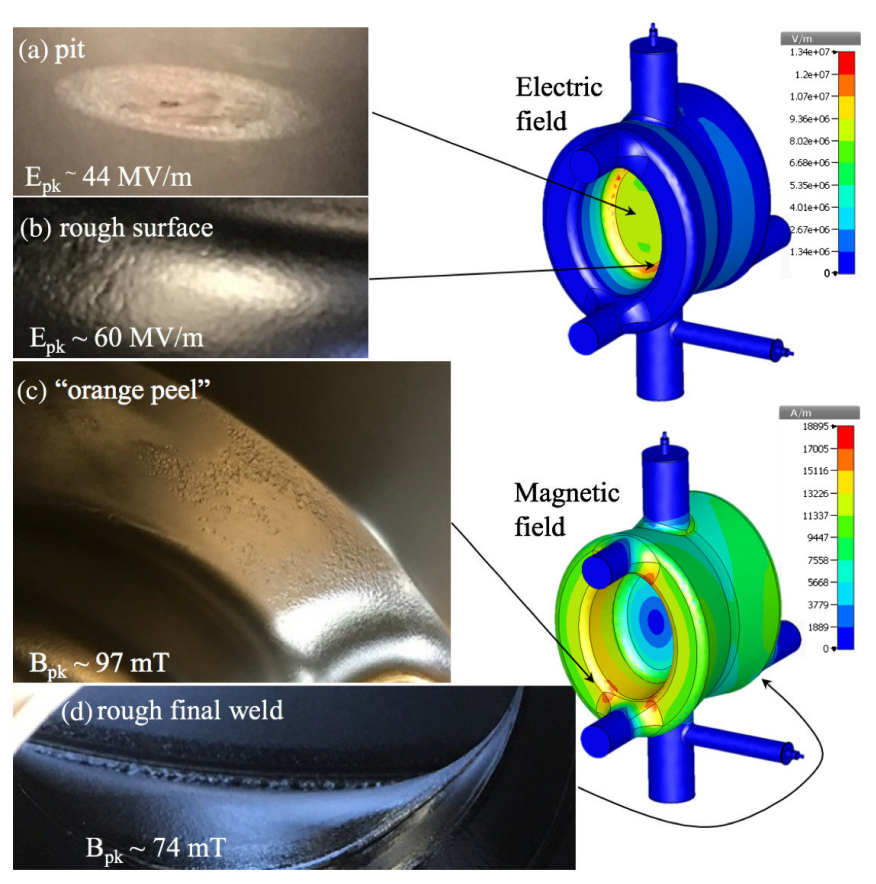

FIG. 9. Surface features found in the rf surface of cavity 1 after bulk BCP. Field values correspond to the quench voltage (5.9 MV).

possible multipacting conditioning and (b) high enough to explore the quench limit in the case of a high-field $Q$ slope.

The pickup probe was inserted into its port for an external $Q$ of about $1 \times 10^{12}$. Both input and pickup probes were hooks made of copper. The DN100 and DN63 stainless-steel flanges had all been coated at CERN with a thin film of niobium, including the zero-length flanges hosting the coupler feedthroughs. Coating the stainlesssteel flanges with a niobium film increased the intrinsic $Q$ of these components by 6 orders of magnitude. rf-seal copper gaskets were used in every DN100 and DN63 flange connection to further reduce power losses [43].

Cavity 1 and cavity 2 were tested in the vertical orientation. The cavities had a stiffening frame to prevent plastic deformation during cooldown, as shown in Fig. 10. The frame was made of titanium. The stiffening frame held the central pin of both capacitive plates. The Lorentz force detuning was $-553 \mathrm{~Hz} /(\mathrm{MV})^{2}$ for cavity $1(6 \mathrm{kHz}$ at the nominal deflecting voltage). The pressure sensitivity, measured at low field during warmup, was $-743 \mathrm{~Hz} / \mathrm{mbar}$. Lower-frequency sensitivities are expected for the dressed SPS-series DQW cavities. The helium vessel and tuning system of the SPS-series DQW cavities [38] should stiffen cavity ports (inductive region) and plates (capacitive region) more than the stiffening frame used for cryogenic rf tests.

\section{A. Cavity 1}

Cavity 1 was cold tested in February 2017. This was the first cryogenic rf test of a DQW SPS-series cavity.

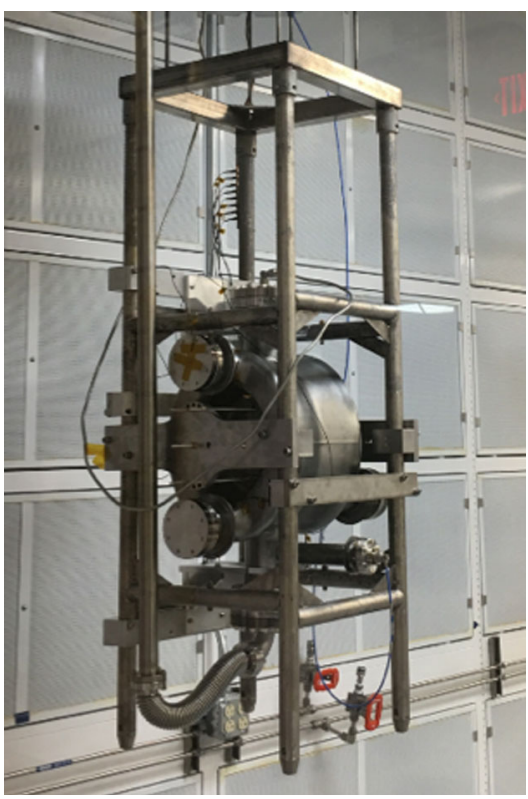

FIG. 10. Stiffening frame for DQW cold tests at Jefferson Lab.

The main goals of the test were to (i) determine the quench limit of the cavity at $2 \mathrm{~K}$ and (ii) provide data for future cavity commissioning and operation on multipacting, field emission, and heat loads.

The intrinsic $Q$ of the whole cavity assembly was $(9.8 \pm$ $1.0) \times 10^{9}$ at the low field, $(6.5 \pm 0.6) \times 10^{9}$ at the nominal voltage level, and $(2.29 \pm 0.17) \times 10^{9}$ at the quench field. The cavity reached the maximum deflecting voltage of $(5.9 \pm 0.4)$ MV before quenching, as shown in Fig. 11. Errors for these quantities were estimated from the expressions detailed in Ref. [44]. The calculations assumed a $7 \%$ error coming from cable and reference power meter calibrations and $2 \%$ error from power meter linearity. The voltage standing-wave ratio of the rf system setup was 1.12 .

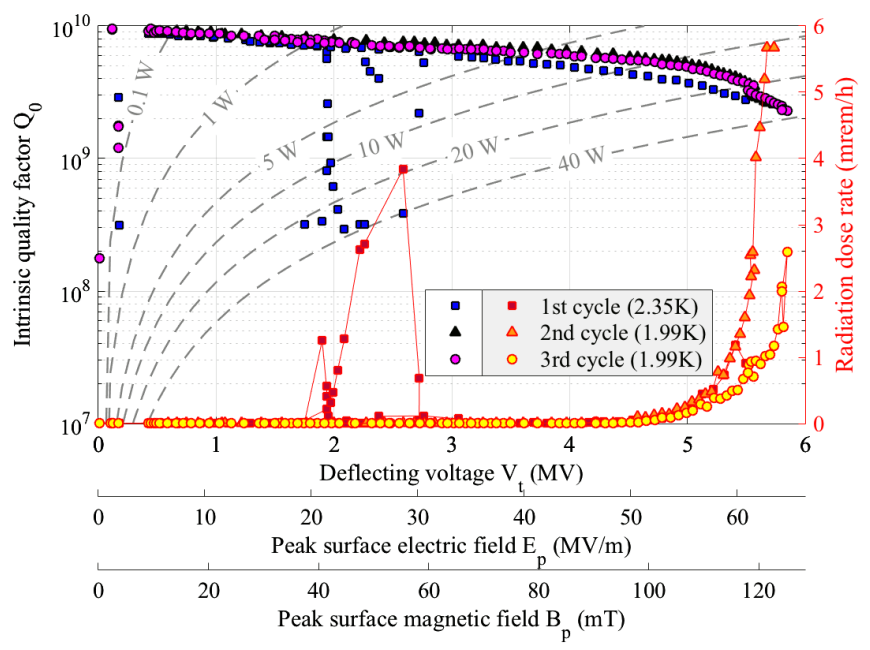

FIG. 11. $Q_{0}-V_{t}$ curves and radiation measured during the cold test of cavity 1 at Jefferson Lab. 
At the nominal deflecting voltage (3.4 MV), less than $5 \mathrm{~W}$ were dissipated in the cavity. The cavity quenched at 5.9 MV deflecting voltage, when $32 \mathrm{~J}$ were stored in the cavity volume with a total if dynamic loss of $35 \mathrm{~W}$. Isopower contours are drawn in Fig. 11. At the quench voltage (5.9 MV), we estimate that around $191 \mathrm{~mW}$ were dissipated in the copper input probe, $20 \mathrm{~mW}$ in the copper gaskets, and $40 \mathrm{~mW}$ in the uncoated regions of the beam port flanges. The losses in other components (pickup probe, coated flanges) were less than $0.13 \mathrm{~mW}$. The cavity walls dissipated about $35 \mathrm{~W}$. The intrinsic $Q$ of assembly components (flanges, gaskets, and test probes, without the contribution from the cavity) was $3.2 \times 10^{11}$. The surface resistance values used to calculate the dissipated power in each component were $1 \mathrm{~m} \Omega$ for copper (the value accounts for the anomalous skin effect suffered by good conductors at cryogenic temperatures and $30 \%$ additional losses due to surface roughness), $30 \mathrm{~m} \Omega$ for stainless steel, and $20 \mathrm{n} \Omega$ for niobium film on the blank flanges. The calculation assumed a constant surface resistance value, independent of the temperature increase due to the Joule effect. The surface resistance of the cavity is estimated to be $9 \mathrm{n} \Omega$ from the calculated geometry factor and the measured intrinsic $Q$ at low fields.

The BCS surface resistance for a niobium cavity operating at a frequency $f$ smaller than $10^{12} \mathrm{~Hz}$ and at a temperature $T$ lower than half of the critical temperature $T_{c}$ is given by the fitted expression [45]

$$
R_{\mathrm{BCS}}(\Omega)=2 \times 10^{-4} \frac{1}{T}\left(\frac{f}{1.5}\right)^{2} \exp \left(-\frac{17.67}{T}\right) .
$$

Using this expression, the BCS surface resistance contributes about $1 \mathrm{n} \Omega$ to the estimated surface resistance mentioned above. On the other hand, the contribution of the residual magnetic field to the surface resistance of the cavity is given by [45]

$$
R_{\text {mag }}=0.3(\mathrm{n} \Omega) H_{\text {ext }}(\mathrm{mOe}) \sqrt{f(\mathrm{GHz})},
$$

where $H_{\text {ext }}$ is the residual magnetic field and $f$ is the operating frequency of the cavity. The residual magnetic field in the Dewar was $5.3 \mathrm{mG}$ maximum, thus contributing with $1 \mathrm{n} \Omega$ to the total surface resistance of the cavity.

Both POP and SPS cavities exhibited $Q$ switches at various field levels. $Q$ switches had been observed in POP cavity cold tests even after surface reprocessing [46].

Eight Cernox $^{\mathrm{TM}}$ temperature sensors were used to monitor the temperature at critical locations (high field regions, coated flanges, regions with difficult access for cleaning, and the closest point on the cavity to the vaporliquid helium interface). The location of the temperature sensors is shown in Fig. 12. Temperature sensor 2-located on top of the FPC port blank flange in the U.S. prototype number 1-registered an abrupt temperature increase
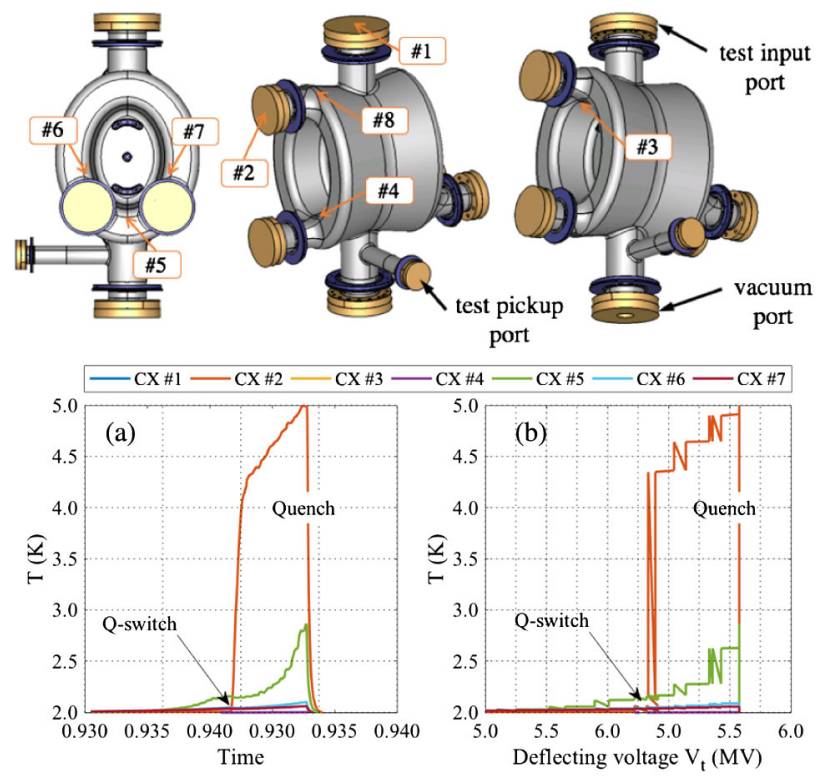

FIG. 12. (Above) Temperature sensors locations for a cold test of cavity 1 and a first cold test of cavity 2. (Below) Signal recorded from temperature sensors 1-7 during a cold test of cavity 1: (a) temperature versus time; (b) temperature versus deflecting voltage.

when the cavity had reached a deflecting voltage of $(5.6 \pm 0.2) \mathrm{MV}$. The $Q$ switch was found at the same voltage level. We suspect that a defect in the niobium coating may be related to the observed $Q$ switches.

Temperature sensor 5 registered a slow temperature increase starting at 5.0 MV deflecting voltage, reaching almost $3 \mathrm{~K}$ at quench. Temperature sensor 5 was located between the two HOM ports in the bottom subassembly. This location corresponds to the region with the largest peak magnetic field. The magnetic field in the region is $72.8 \mathrm{mT}$ at $3.4 \mathrm{MV}$ and $106 \mathrm{mT}$ at 5.0 MV deflecting voltage. The magnetic field is as high as $125 \mathrm{mT}$ at quench voltage in the same region, in contrast to the $0.35 \mathrm{mT}$ reached at temperature sensor 2 . These field values are those calculated for the ideal cavity model. Temperature sensors 6 and 7, located at the other sides of the HOM ports in the bottom subassembly, also registered an increase in temperature but to a lesser extent than temperature sensor 5 . Temperature sensor 8 did not work appropriately during the test. Signals registered by the temperature sensors are shown in Fig. 12.

The limited time to test this cavity did not allow running in a pulsed mode, to investigate if the quench was thermal or magnetic. The FPC port is the shortest port on top of the high magnetic field region of the cavity. The power dissipated in the $\mathrm{Nb}$-coated stainless-steel flange of the FPC port was estimated as $3.38 \times 10^{-6} \mathrm{~W}$ at the quench field. If the niobium film quenched or was damaged, the losses could be as much as 6 orders of magnitude higher and induce a thermal quench. This possibility seems plausible. Temperature sensor 2 registered a temperature 
of $5 \mathrm{~K}$ right before cavity quench. The temperature difference between the surface and bath is larger than the bath temperature itself (at $2 \mathrm{~K}$ ). In this regime, the heat transfer from the surface into He-II is given by

$$
\dot{q}=\alpha\left(T_{s}^{m}-T_{b}^{m}\right),
$$

where $\dot{q}$ is the heat flux per unit of area, $T_{s}$ is the surface temperature, and $T_{b}$ is the bath temperature. The Kapitza coefficient $\alpha$ and the power-law exponent $m$ are empirical and dependent on the material, temperature, and surface status. Hereafter, the fitted values of $\alpha=22.4 \mathrm{~W} / \mathrm{m}^{2} / \mathrm{K}^{m}$ and $m=2.72$ for polished SS304L are used [47]. The necessary heat to increase the surface temperature is generated by the Joule effect. The power dissipated per unit of area is calculated from

$$
\dot{q}=\frac{1}{2} R_{s}\left|J_{s}\right|^{2},
$$

where $R_{s}$ is the rf surface resistance of the flange surface exposed to rf and $J_{s}$ is the surface current in the flange at the quench field $\left(J_{s}=480 \mathrm{~A} / \mathrm{m}\right)$. The $R_{s}$ thereby calculated is about a hundredth of an Ohm, closer to the surface resistance of stainless steel than of superconducting niobium and thus supporting the hypothesis of a thermal quench.

On the other hand, the peak surface magnetic field in some regions of the cavity was $125 \mathrm{mT}$ when the cavity quenched, so a magnetic quench cannot be dismissed. In any case, cavities installed in SPS and the LHC will not have blank flanges in their ports, so the thermal quench scenario is less likely and higher deflecting voltages could potentially be reached.

Two main multipacting regions were found during the cold test. The first region appeared for deflecting voltages lower than 0.3 MV. The POP cavity also showed this multipacting region and was easily conditioned during the cold tests in 2013 with an adjustable input coupler. Multipacting did not come back once the POP cavity was conditioned. The limited time available to test the present cavity with a fixed coupler did not allow, however, a full conditioning.

Simulations predicted weak multipacting signatures in the blending of the small ports for deflecting voltages between 1.7 and 3.0 MV. The impact energy of the electrons multipacting in this region is below $100 \mathrm{eV}$, for which the SEY coefficient is barely larger than 1 . This multipacting band actually appeared between 1.7 and 2.7 MV during the 1st cycle of the cavity test and was easily conditioned in a couple of hours with less than $100 \mathrm{~W}$ of rf input power (it does not come back in the $2 \mathrm{nd}$ and 3rd cycles). However, the conditioning led to high radiation levels and large $Q$ degradation, as seen in Fig. 11. According to simulations, the impact energy of electrons should be small, not enough to make penetrating radiation.
In addition, the SEY coefficient is small, so very few electrons are actually stripped from the metal. Therefore, for such a large radiation level and $Q$ degradation, the number of multipacting electrons must also be large and their energy high enough to generate penetrating radiation electron. A "spray" of electrons in the wrong phase or initial impact site may travel to cavity regions with higher fields where electrons get accelerated. The impact of these electrons against the cavity walls would then lead to the high radiation observed during the 1 st cycle of the test between 1.7 and $2.7 \mathrm{MV}$.

Field emission became significant for peak surface electric fields above $45 \mathrm{MV} / \mathrm{m}$, at $4.1 \mathrm{MV}$ deflecting voltage, already larger than nominal. Such a high-field onset reflects the good surface quality of the cavity. A reduction of the radiation emitted from the 2 nd to the 3 rd cycle suggests that the cavity conditioned while measurements were being performed. The maximum peak surface field reached in the cavity during the cold test was about $65 \mathrm{MV} / \mathrm{m}$. The radiation monitor was located inside the shielding hatch, near the Dewar top plate.

\section{B. Cavity 2}

Cavity 2 underwent its first cold test in June 2017. The main goal of this test was to confirm the results obtained with cavity 1 . The cavity presented an intrinsic $Q$ of $(9.2 \pm 1.1) \times 10^{9}$ at low fields and reached up to $(5.3 \pm$ $0.3)$ MV deflecting voltage. Several multipacting bands were found and successfully processed in about $1 \mathrm{hr}$. The dissipated power was $4 \mathrm{~W}$ at a nominal deflecting voltage of 3.4 MV. Field emission started at 2.4 MV and led to a pronounced $Q$ slope. The cavity did not quench; the operation was interrupted due to administrative power limitations. Temperature sensors again identified a temperature increase in the region between the two $45^{\circ} \mathrm{HOM}$ ports when voltages were above $5 \mathrm{MV}$.

The cavity surface was treated again (light BCP of $18 \mu \mathrm{m}$ plus HPR) with the aim of increasing the voltage of the field-emission onset and attempting to reach higher deflecting voltages. The second cold test of cavity 2, also conducted at Jefferson Lab, was held in September 2017. The temperature sensors' distribution for the September test was changed according to Fig. 13. The inspection of cavity 2 before bulk BCP found some spatter onto and around the two last welds. These welds were also rough. Figure 14 shows a selection of the features found in the rf surface of cavity 2. Temperature sensors 6 and 8 were placed on top of the location where spatter was observed. Temperature sensors 4, 5, and 7 monitored local and global high magnetic field regions. Temperature sensor 3 was placed where previous tests had registered a temperature increase. Temperature sensor 2 monitored the zero-length flange in the vacuum port where the niobium coating presented a scratch. Last, temperature sensor 1 monitored the saturated helium bath level above the cavity. 

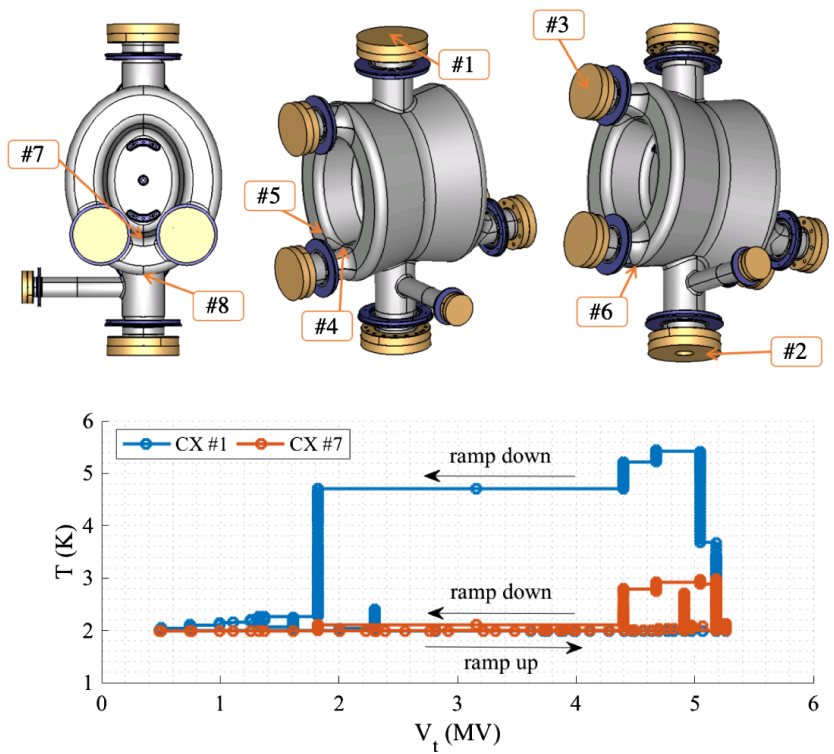

FIG. 13. Test of cavity 2 in September 2017: thermosensor location (above) and signal recorded from thermosensors (below).

The cavity test found again the low-field (below $0.2 \mathrm{MV}$ ), hard multipacting predicted by ACE3P. The cavity was conditioned for $1.5 \mathrm{hr}$ at $10-20 \mathrm{~W}$ input power before the first breakthrough. Quenches would later cause the cavity to fall into this low-field multipacting region for about $30 \mathrm{~min}$. Soft multipacting regions were found between 1.1 and 3.0 MV and at 4.5 MV, in perfect agreement with CST and ACE3P simulations. About $35 \mathrm{~W}$ maximum were used to go through multipacting.

The $Q-V_{t}$ curves for all the bare cavity tests of SPS-series DQW cavities fabricated in the U.S. are displayed together in Fig. 15. In the September test, cavity 2 quenched at $(5.3 \pm 0.2) \mathrm{MV}$ during $\mathrm{cw}$ operation.

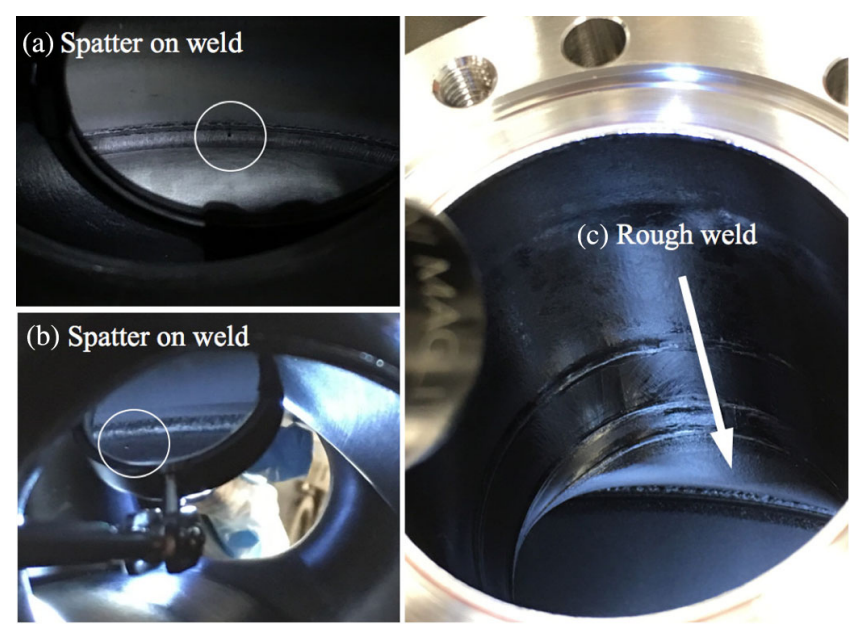

FIG. 14. Surface features in cavity 2 before bulk BCP. Electric and magnetic fields on the weld area are, respectively, $2 \mathrm{MV} / \mathrm{m}$ and $65 \mathrm{mT}$ maximum, at 5.3 MV deflecting voltage.

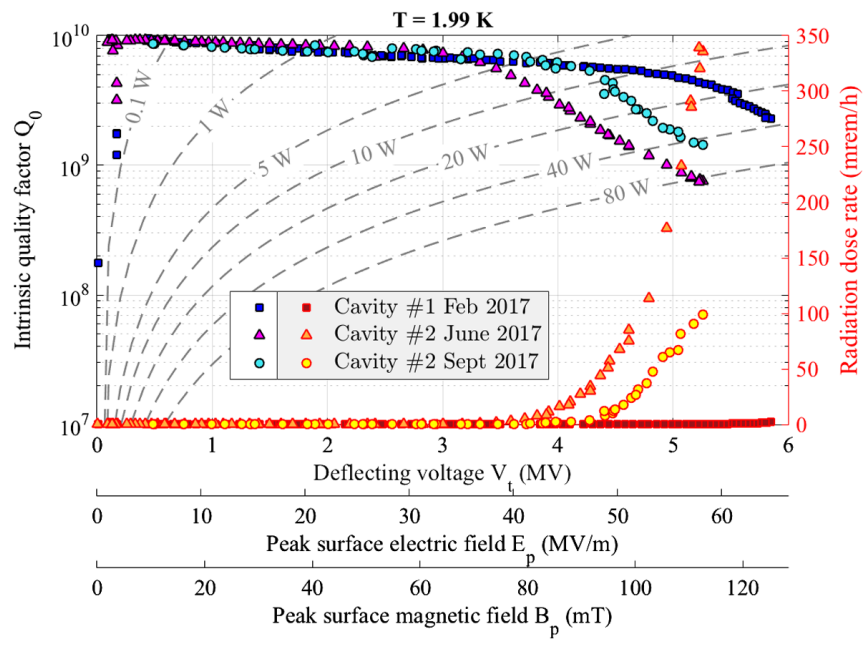

FIG. 15. $Q-V_{t}$ curves measured for the two U.S.-produced SPS-series DQW cavities.

The same quench limit was found during operation with 1.4-s-long rf pulses at $0.3 \mathrm{~Hz}$ repetition rate. This finding suggests that the cavity voltage limitation is due to a magnetic quench.

The field-emission onset, and consequently the pronounced $Q$ slope, appeared at higher voltages (2.8 MV) than for the June test. The improvement is attributed to the light $\mathrm{BCP}$ and HPR performed between tests. The performance of cavities 1 and 2, in terms of voltage, is comparable when accounting for measurement errors. In terms of efficiency, however, the performance of cavity 2 is not comparable to cavity 1 due to earlier field emission (evidenced by a much lower $Q$ and higher radiation at voltages above $4 \mathrm{MV}$ ). Still, both cavities satisfy the heat load requirement, with a power dissipation below $5 \mathrm{~W}$ per cavity during operation at the nominal voltage.

Only temperature sensors 1 and 7 registered a signal associated to the quench. Other temperature sensors simply followed the bath temperature, indicating that they were either in a region where temperature did not increase or detached from the cavity surface. After quench, as fields ramped down in the cavity, temperature sensors 1 and 7 monitored high temperatures (see Fig. 13). Temperature sensor 1 reached a higher temperature and stayed hot for a longer time after quench than temperature sensor 7. The longer thermal path from the bath to the niobium coating in the short beam port flange would explain why it took a longer time for point 7 to recover, as the niobium film needs to become superconducting again after a quench.

\section{DISCUSSION}

Cold tests of the two DQW SPS-series cavities fabricated in the U.S. greatly surpassed the nominal deflecting voltage requested per cavity (3.4 MV), going beyond the ultimate deflecting voltage (5 MV) up to 5.3-5.9 MV. Comparable 
TABLE III. Summary of cavity test performances.

\begin{tabular}{|c|c|c|c|c|}
\hline Magnitude & & $\begin{array}{c}\text { Cavity } 1 \\
\text { (February 2017) }\end{array}$ & $\begin{array}{c}\text { Cavity } 2 \\
\text { (September 2017) }\end{array}$ & Unit \\
\hline Maximum deflecting voltage & $V_{t}^{\max }$ & 5.9 & 5.3 & MV \\
\hline Maximum stored energy & $U^{\max }$ & 32 & 25 & $\mathrm{~J}$ \\
\hline Maximum dissipated power ${ }^{\mathrm{a}}$ & $P_{0}^{\max }$ & 35 & 45 & W \\
\hline Maximum peak surface electric field & $E_{p}^{\max }$ & 64.7 & 58.2 & $\mathrm{MV} / \mathrm{m}$ \\
\hline Maximum peak surface magnetic field & $B_{p}^{\max }$ & 125.2 & 112.6 & $\mathrm{mT}$ \\
\hline Intrinsic $Q$ at nominal deflecting voltage & $Q_{0}^{\text {nom }}$ & $6.5 \times 10^{9}$ & $7.2 \times 10^{9}$ & \\
\hline Intrinsic $Q$ at maximum field & $Q_{0}^{\max }$ & $2.3 \times 10^{9}$ & $1.4 \times 10^{9}$ & \\
\hline Field-emission onset & $V_{t}^{\mathrm{FE}}$ & 4.1 & 2.8 & MV \\
\hline
\end{tabular}

${ }^{\mathrm{a}}$ Includes losses in couplers, flanges, and gaskets.

results were reached in 2017 by an RFD prototype [48]. Table III summarizes the cryogenic rf performances of the two bare cavities. The successful results demonstrate the maturity of the DQW cavity rf design. Remarkably, the maximum peak surface fields reached with cavity 1 are close to those in a typical TESLA-type cavity operating at $30 \mathrm{MV}$ accelerating voltage (60 MV/m and $128 \mathrm{mT}$ ) [31]. The maximum magnetic field of cavity 1 is comparable to the highest values reached by other SRF cavities that followed a BCP-based surface treatment [49-52]. The dynamic cryogenic load of the two DQW SPS-series prototypes meets the specification for tests in SPS and operation in the LHC. In addition, the test results demonstrate the possibility of manufacturing by industry and the sufficiency of standard SRF surface treatments to reach the required specifications for the HL-LHC. The experience acquired with the design, fabrication, and testing of these cavities will serve as a guideline for the development of the eRHIC crab cavities, also based on the DQW concept.

The test of cavity 1 exceeded the requested nominal voltage $(3.4 \mathrm{MV})$ by more than $70 \%$ and the ultimate voltage $(5.0 \mathrm{MV})$ by more than $15 \%$. The current HL-LHC program envisages the installation of two crab cavities per side per beam per IP (from the initial four cavities per side per beam per IP). Whereas dressed cavities tend to show worse performances than bare cavities, the test results bring some optimism that $10 \mathrm{MV}$ deflecting voltage can be provided by only two cavities, while the project envisioned four cavities at first. Recent DQW SPS-series cavity tests with a HOM filter have reached 4.7 MV before quench at $2 \mathrm{~K}$ and $\mathrm{cw}$ operation. Investigations are now in place to push the cavity and filter performance.

The performance of the two bare DQW SPS-series prototypes was limited by quench. Quenches were accompanied by a large temperature increase in the highest magnetic field region of the cavities and in one of the $\mathrm{Nb}$-coated flanges diametrically opposed to the highest magnetic field region (the short small port in cavity 1 and the short beam port in cavity 2). Further tests would be required to determine if the quenches are magnetic or thermal. The maximum achievable voltage may be limited by the use of niobium-coated flanges. Future tests intended to explore the ultimate performance of the DQW cavities should consider the use of niobium extension tubes in replacement of the niobium-coated flanges.

The limited duration of the tests did not allow for full processing of the multipacting. The DQW cavities will be well overcoupled during the SPS test in 2018 (the fundamental power coupler for the SPS test is designed for an external $Q$ of around $5 \times 10^{5}$ ), so enough power will be available to process multipacting. Additional tests of bare and dressed cavities will help determining if the origin of $Q$ switches is coated flanges or the input probe itself.

The surface features found after the last BCP did not seem to jeopardize the performances of cavity 1 . On the other hand, cavity 2 did suffer a pronounced $Q$ slope accompanied by large radiation. Future studies will evaluate the cavity performances after electropolishing. Electropolishing provides smoother surfaces than BCP [53]. A reduction of the surface roughness may push the start of the field emission to higher deflecting voltages.

In the future, we plan to study how nitrogen infusion may impact the cavity performances. The $Q$ slope becomes more acute for fields beyond the nominal deflecting voltage. Nitrogen infusion has twice enabled the stateof-the-art $Q$ at $2 \mathrm{~K}$ and a field of $190 \mathrm{mT}$ to be reached in cold tests of $1.3 \mathrm{GHz}$ bulk niobium cavities [54]. The effects of nitrogen infusion in cavities operating at $400 \mathrm{MHz}$ are currently unknown. The nitrogen infusion treatment may have a limited impact on the $400 \mathrm{MHz}$ DQW cavity, though. First, recent studies found that lowfrequency cavities did not show the characteristic $R_{\mathrm{BCS}}$ reversal found in $1.3 \mathrm{GHz}$ cavities [55]. Second, the maximum $Q_{0}$ of the DQW cavities is driven by the intrinsic residual surface resistance. Studies of flux trapping in the DQW cavity geometry may provide some insight on better cooldown schemes to reduce the magnetic component of the residual surface resistance. The impact on the cavity performance also might be limited for a cavity with such a low frequency [56].

The SPS DQW cavity design satisfies the requirements to be installed in a cryomodule for operation in the LHC. 
However, the fabrication, preparation, and testing of several DQW cavities have shed light on possible design improvements that could boost the cavity performance and/or reduce cavity fabrication time and cost. One first improvement would consist in lengthening the shortest beam pipe to dispense with the niobium coating of the pipe flange. These possibilities are now being studied prior to the production of the LHC-series cavities.

\section{ACKNOWLEDGMENTS}

The authors are thankful to Jingsong Wang (CST) and Chien-Ih Pai (BNL) for technical support to run simulations. Thanks go to Dave Hall and Sam Baurac at Niowave for the careful machining work on the subassembly thinning cuts and $\mathrm{rf}$ tuning measurements. We are also grateful to Alick MacPherson (CERN) for providing the niobium coated flanges used in these tests. Work was partly supported by U.S. DOE through Brookhaven Science Associates LLC under Contract No. DE-AC02-98CH10886 and the U.S. LHC Accelerator Research Program (LARP). This research used resources of the National Energy Research Scientific Computing Center, which is as well supported by U.S. DOE under Contract No. DE-AC02-05CH11231. The research also received support from the EU FP7 HiLumi LHC, Grant Agreement No. 284404. Cavity fabrication at Niowave Inc. was supported by DOE SBIR Grant No. DE-SC0007519.

[1] R. Calaga, E. Jensen, G. Burt, and A. Ratti, Crab cavity development, in The High Luminosity Large Hadron Collider, edited by O. Brüning and L. Rossi (World Scientific, Singapore, 2015), Vol. 22, pp. 137-156.

[2] R. Calaga, LHC crab cavities, talk in DOE LARP Review (Fermilab, Batavia, 2012).

[3] S. U. De Silva and J. R. Delayen, Design evolution and properties of superconducting parallel-bar rf-dipole deflecting and crabbing cavities, Phys. Rev. Accel. Beams 16, 012004 (2013).

[4] S. U. De Silva and J. R. Delayen, Cryogenic test of a proofof-principle superconducting rf-dipole deflecting and crabbing cavity, Phys. Rev. Accel. Beams 16, 082001 (2013).

[5] B. Hall, G. Burt, R. Apsimon, C. J. Lingwood, A. Tutte, A. Grudiev, A. Macpherson, M. Navarro-Tapia, R. Calaga, K. G. Hernández-Chahín, R. B. Appleby, and P. Goudket, Design and testing of a four rod crab cavity for High Luminosity LHC, Phys. Rev. Accel. Beams 20, 012001 (2017).

[6] High-Luminosity Large Hadron Collider (HL-LHC) Technical Design Report v.0.1, edited by G. Apollinari, I. Béjar Alonso, O. Brüning, P. Fessia, M. Lamont, L. Rossi, and L. Tavian CERN Yellow Reports: Monographs, Vol. 4 (CERN, Geneva, 2017), http://dx.doi.org/10.23731/ CYRM-2017-004.

[7] A. Arno et al. (eRHIC Collaboration team), eRHIC PreConceptual Design Report, Brookhaven National Laboratory Report, edited by J. Beebe-Wang (to be published).
[8] S. Sosa, Crab cavity requirements for the Jefferson Lab electron-ion collider, in Proceedings of the APS April Meeting 2018, Columbus, Ohio, 2018 (unpublished).

[9] S. Verdú-Andrés, I. Ben-Zvi, Q. Wu, and R. Calaga, Crab cavity systems for future colliders, in Proceedings of the 8th International Particle Accelerator Conference (IPAC'17) (JACoW, Copenhagen, 2017), pp. 2474-2477.

[10] Y. Funakoshi (KEKB commissioning group), Operational experiencewith crab cavities at KEKB, in Proceedings of the ICFA Mini-Workshop on Beam-Beam Effects in Hadron Colliders, edited by W. Herr and G. Papotti (CERN, Geneva, 2014), pp. 27-36.

[11] C. Zanoni et al., The crab cavities cryomodule for SPS test, J. Phys. Conf. Ser. 874, 012092 (2017).

[12] K. Hosoyama, K. Hara, A. Kabe, Y. Kojima, Y. Morita, H. Nakai, K. Saito, T. Furuya, K. Akai, H. Hattori, and S. Imatake, Crab cavity for $\mathrm{KEKB}$, in Proceedings of the Workshop on RF Superconductivity (SRF'95) (JACoW, Gif-sur-Yvette, 1995), pp. 671-675.

[13] D. Alesini, G. Di Pirro, L. Ficcadenti, A. Mostacci, L. Palumbo, J. Rosenzweig, and C. Vaccarezza, RF deflector design and measurements for the longitudinal and transverse phase space characterization at SPARC, Nucl. Instrum. Methods Phys. Res., Sect. A 568, 488 (2006).

[14] V. Shemelin and S. Belomestnykh, RF design of the deflecting cavity for beam diagnostics in ERL injector, CLASSE Cornell Report No. ERL 07-2, 2007.

[15] G. Burt, P. K. Ambattu, A. C. Dexter, T. Abram, V. Dolgashev, S. Tantawi, and R. M. Jones, X-band crab cavities for the CLIC beam delivery system, Report No. SLAC-PUB-14769, 2008.

[16] Z. Li, L. Xiao, C. Ng, and T. Markiewicz, Compact 400$\mathrm{MHz}$ half-wave spoke resonator crab cavity for the LHC upgrade, Report No. SLAC-PUB-14163, 2010.

[17] C. Hovater, G. Arnold, J. Fugitt, L. Harwood, R. Kazimi, G. Lahti, J. Mammosser, R. Nelson, C. Piller, and L. Turlington, The CEBAF RF separator, in Proceedings of the International Linear Accelerator Conference (LINAC'96) (JACoW, Geneva, 1996), pp. 77-79.

[18] R. Calaga, LHC upgrade and crab cavities, in Proceedings of the ICFA Deflecting Cavity Workshop, Lanzhou, 2012 (unpublished).

[19] Q. Wu, Double quarterwave crab cavity, in Proceedings of LARP CM18/HiLumi LHC meeting, Fermilab, Batavia, 2012 (unpublished).

[20] I. Ben-Zvi, 1/4-wave LHC crab cavity, in Proceedings of the 5th LHC Crab Cavity Workshop (LHC-CC11), CERN, Geneva, 2011 (unpublished).

[21] B. Hall, Review of options for crab cavities in LHC, Proceedings of the TESLA Collaboration Meeting (TTC'12), Jefferson Lab, Newport News, 2012 (unpublished).

[22] R. Calaga, S. Belomestnykh, I. Ben-Zvi, and Q. Wu, A quarterwave design for crab crossing in the LHC, in Proceedings of the 3rd International Particle Accelerator Conference (IPAC'12), New Orleans, LA, 2012 (IEEE, Piscataway, 2012), pp. 2260-2262.

[23] R. Calaga, S. Belomestnykh, I. Ben-Zvi, J. Skaritka, Q. $\mathrm{Wu}$, and B. Xiao, A double quarter wave deflecting cavity for the LHC, in Proceedings of the 4th International 
Particle Accelerator Conference (IPAC'13), Shanghai, China, 2013 (JACoW, Shanghai, 2013), pp. 2408-2410.

[24] L. Rossi, Outcome of the cost and schedule review and changes to the HL-LHC baseline, in Proceedings of the 6th HL-LHC Collaboration Meeting, Paris, 2016 (unpublished).

[25] B. P. Xiao, L. Alberty, S. Belomestnykh, I. Ben-Zvi, R. Calaga, C. Cullen, O. Capatina, L. Hammons, Z. Li, C. Marques, J. Skaritka, S. Verdú-Andrés, and Q. Wu, Design, prototyping and testing of a compact superconducting double quarter wave crab cavity, Phys. Rev. Accel. Beams 18, 041004 (2015).

[26] P. Baudrenghien, K. Brodzinski, R. Calaga, O. Capatina, E. Jensen, A. Macpherson, E. Montesinos, and V. Parma, Functional specifications of the LHC prototype crab cavity system, CERN Report No. CERN-ACC-NOTE-2013-003, 2013 (unpublished).

[27] B. P. Xiao, S. Belomestnykh, I. Ben-Zvi, R. Calaga, S. Verdú-Andrés, and Q. Wu, Compact higher order mode filter for crab cavities in the Large Hadron Collider, in Proceedings of the 16th International Conference on $R F$ Superconductivity (SRF'13) (JACoW, Paris, 2013), pp. 1006-1008.

[28] Q. Wu, Double quarter wave crab cavity design, in LHC Crab Cryostat Integration and Planning Meeting, Fermilab, Batavia, 2013 (unpublished).

[29] N. Biancacci, B. Salvant, E. Métral, and K. Li, The HL-LHC impedance model and aspects of beam stability, in Proceedings of the 7th International Particle Accelerator Conference (IPAC'16) (JACoW, Busan, 2016), pp. 606-609.

[30] S. Verdú-Andrés, J. Skaritka, Q. Wu, B. P. Xiao, S. Belomestnykh, I. Ben-Zvi, R. Calaga, and Z. Li, Optimization of the double quarter wave crab cavity prototype for testing at SPS, in Proceedings of the 16th International Conference on RF Superconductivity (SRF'13) (JACoW, Paris, 2013), pp. 995-997.

[31] B. Aune et al., Superconducting TESLA cavities, Phys. Rev. Accel. Beams 3, 092001 (2000).

[32] H. Padamsee, RF Superconductivity, 1st ed. (Wiley-VCH, Weinheim, 2009).

[33] Advanced Computational Electromagnetic Simulation Suite ACE3P, SLAC National Accelerator Laboratory, Menlo Park, CA, 2013.

[34] Computer Simulation Technology CST, CST AG, Darmstadt, Germany, 2015.

[35] R. Calder, G. Dominichini, and N. Hilleret, Influence of various vacuum surface treatments on the secondary electron yield of niobium, Nucl. Instrum. Methods Phys. Res., Sect. B 13, 631 (1986).

[36] B. P. Xiao, S. Belomestnykh, I. Ben-Zvi, G. Burt, R. Calaga, O. Capatina, B. Hall, T. Jones, J. Skaritka, S. Verdú-Andrés, and Q. Wu, Higher order mode filter design for double quarter wave crab cavity for the LHC high luminosity upgrade, in Proceedings of the 6th International Particle Accelerator Conference (IPAC'15) (JACoW, Richmond, 2015), pp. 3627-3629.

[37] N. Kuder, DQWdressed cavity strength assessment, CERN Report EDMS No. 1549819, 2016 (unpublished).

[38] C. Zanoni et al., Design of dressed crab cavities for the HL-LHC upgrade, in Proceedings of the 17th International
Conference on RF Superconductivity (SRF'15) (JACoW, Whistler, 2015), pp. 1284-1288.

[39] J. Sears and B. Clasby, Developments in electron beam welding of niobium cavities, in Proceedings of the 12th International Workshop on RF Superconductivity (SRF'05) (Cornell University, Ithaca, 2005), paper THP12.

[40] Engineering simulation and 3D design software ANSYS, ANSYS, Inc., Canonsburg, PA, 2014.

[41] S. Verdú-Andrés, S. Baurac, C. Boulware, W. Clemens, T. Grimm, A. E. McEwen, H. Park, A. Ratti, J. Skaritka, Q. $\mathrm{Wu}$, and J. Yancey, Trim tuning of SPS-series DQW crab cavity prototypes, in Proceedings of the 8th International Particle Accelerator Conference (IPAC'17) (JACoW, Copenhagen, 2017), pp. 1187-1189.

[42] S. Verdú-Andrés, DQW Cavity MIP, CERN Report EDMS No. 1569808 (unpublished).

[43] A. Castilla, N. Shipman, and S. Verdú-Andrés, CRAB DQW SPS test antennas, CERN Report EDMS No. 1745787 (unpublished).

[44] T. Powers, Practical aspects of SRF cavity testing and operations, in Tutorials of the 18th International Conference on RF Superconductivity (SRF'17), Lanzhou, 2017 (unpublished).

[45] H. Padamsee, J. Knobloch, and T. Hays, RF Superconductivity for Accelerators, 2nd ed. (Wiley-VCH, Weinheim, 2011).

[46] K. G. Hernández-Chahín, G. Burt, C. Jarrige, S. De Silva, A. MacPherson, M. Navarro-Tapia, R. Torres-Sánchez, A. Tutte, and S. Verdú-Andrés, Performance evaluation of HL-LHC crab cavity prototypes in a CERN vertical test cryostat, in Proceedings of the 17th International Conference on RF Superconductivity (SRF'15) (JACoW, Whistler, 2015), pp. 1210-1214.

[47] M. Taneda, T. Miki, and T. Ohtani, The Kapitza conductance of PVF-coated copper and some materials used for superconducting magnets, Cryogenics 32, 479 (1992).

[48] S. U. De Silva, H. Park, J. R. Delayen, and Z. Li, RF tests of the RF-Dipole prototype crabbing cavities for LHC high luminosity upgrade, in Proceedings of the 18th International Conference on RF Superconductivity (SRF'17) (JACoW, Lanzhou, 2017), pp. 509-511.

[49] F. He, The R\&D on TEM-type SRF cavities for high-current applications at IHEP, in Proceedings of the 18th International Conference on RF Superconductivity (SRF'17), Lanzhou, 2017 (unpublished).

[50] M. H. Awida, D. Passarelli, P. Berrutti, I. Gonin, S. Kazakov, T. Khabiboulline, J. Holzabauer, T. Nicol, J. Ozelis, M. Parise, Y. Pischalnikov, O. Pronitchev, L. Ristori, G. Romanov, A. Rowe, W. Schappert, D. Sergatskov, N. Solyak, A. Sukanov, and V. P. Yakovlev, Development of low $\beta$ single spoke resonators for the front end of the proton improvement Plan-II at Fermilab, Fermi National Accelerator Laboratory Report No. FERMILABPUB-17-320-TD, 2017 (unpublished).

[51] A. Castilla and J. R. Delayen, Analysis of a $750 \mathrm{MHz}$ SRF dipole cavity, in Proceedings of 17th International Conference on RF Superconductivity (SRF'15) (JACoW, Whistler, 2015), pp. 1200-1204. 
[52] A. Burril, R\&D ERL: 5 cell $704 \mathrm{MHz}$ SRF cavity,, Brookhaven National Laboratory Report No. C-AD/AP/ \#376, 2010 (unpublished).

[53] K. Saito, Surface smoothness for high gradient niobium SC RF cavities, in Proceedings of the 11th Workshop on RF Superconductivity (SRF'03), edited by D. Proch (DESY, Lübeck/Travemünde, 2003), paper ThP15.

[54] A. Grassellino, A. Romanenko, Y. Trenikhina, M. Checchin, M. Martinello, O. S. Melnychuk, S. Chandrasekaran, D. A. Sergatskov, S. Posen, and A. C. Crawford, Unprecedented quality factors at accelerating gradients up to $45 \mathrm{MV} / \mathrm{m}$ in niobium superconducting resonators via low temperature nitrogen infusion, Supercond. Sci. Technol. 30, 094004 (2017).

[55] M. Martinello, Frequency dependence of the BCS surface resistance at high fields, in Proceedings of the TTC Topical Workshop-Pushing Cavity Performance Limits, Fermilab, Batavia, 2017 (unpublished).

[56] M. Checchin, M. Martinello, A. Grassellino, S. Aderhold, S. K. Chandrasekaran, O. Melnychuk, S. Posen, A. Romanenko, and D. A. Sergatskov, Frequency dependence of trapped flux sensitivity in SRF cavities, Appl. Phys. Lett. 112, 072601 (2018). 